\title{
Moving Branes in Presence of the Background Tachyon Fields
}

\author{
Zahra Rezaei and Davoud Kamani \\ Physics Department, Amirkabir University of Technology (Tehran Polytechnic) \\ P.O.Box: 15875-4413, Tehran, Iran \\ e-mails: z.rezaei, kamani@aut.ac.ir
}

\begin{abstract}
We compute the boundary state associated with a moving $\mathrm{D} p$-brane in the presence of the open string tachyon field as a background field. The effect of the tachyon condensation on the boundary state will be discussed. It leads to a boundary state associated with a lower dimensional moving D-brane or a stationary instantonic Dbrane. The former originates from condensation along the spatial directions and the latter comes from the temporal direction of the D-brane's worldvolume. Using the boundary state we also study the interaction amplitude between two arbitrary $\mathrm{D} p_{1}$ and $\mathrm{D} p_{2}$-branes. The long range behavior of the amplitude is investigated which shows an obvious deviation from the conventional form, due to the presence of the background tachyon field.
\end{abstract}

PACS numbers: $11.25 .-\mathrm{w} ; 11.25 . \mathrm{Mj}$

Keywords: Moving branes; Boundary state; Tachyon condensation; Interaction. 


\section{Introduction}

Open string tachyon can be considered as instabilities of the branes, because open strings introduce the quantum excitations of the branes [1]. Tachyon potential has a stationary point where the negative potential energy of the tachyon cancels the tension of the D-brane [2]. This process which is called tachyon condensation ends when the brane has completely disappeared. During the condensation process lower dimensional branes are produced [3, 4].

On the other hand we have the boundary state as a quantum state, which contains closed string states [5]. It can be used to study D-branes. In the other words, a D-brane couples

to all states of the closed string via the boundary state. Thus, we can suppose that the exchange of closed strings between two D-branes is responsible for the branes interaction. For calculating it we can just connect their corresponding boundary states through the closed string propagator. The coherent state method [6] and the path integral approach [7,8] have been used to obtain boundary state. Furthermore, the boundary state in the presence of background fields such as $B_{\mu \nu}$ and $U(1)$ gauge fields in the compact spacetime [9], and in the presence of the tachyon field $[10,11]$ have been investigated.

Apart from the background $U(1)$ gauge field and the open string tachyon field, which are parallel to the brane's worldvolume, the transverse fluctuations of a D-brane also are essential to study it as a dynamical object. Scarcity of this kind of multilateral discussion motivated us to follow this process in this paper in spite of some technical difficulties. Beside, usually in the literature a full brane in the presence of a one-dimensional background tachyon field has been considered and the effect of one-stage condensation on that brane has been studied. But here we study a D-brane with an arbitrary dimension. Therefore, the Dirichlet boundary conditions also will be present. In our set-up the tachyon field has components along all the directions of the D-brane's worldvolume. This tachyon profile leads to various condensations and hence variety of the resulted branes.

In the present article by using the path integral approach we calculate the boundary state corresponding to a moving $\mathrm{D} p$-brane in the presence of a tachyon field. Consequently, we obtain the disk partition function of the closed string. The effect of the tachyon condensation on this partition function will be studied. The condensation is applied along the spatial worldvolume directions and gives a partition function associated with a moving lower dimensional D-brane. Difference with the conventional tachyon condensation (e.g. see [10]) is the presence of a tachyon dependent factor in the resulted partition function. That is, although the brane's dimension decreases, the effect of the tachyon does not remove by condensation. In this process the transverse fluctuations of a $\mathrm{D} p$-brane prevent the normal tachyon con- 
densation from occurring. Applying the condensation along the temporal direction of the $\mathrm{D} p$-brane's worldvolume gives an instantonic stationary brane.

The final goal of the paper is applying the boundary state to obtain interaction amplitude between two moving D-branes and study its behavior for large distances of the branes. We observe that due to the inclusion of the open string tachyon background (which is equivalent to consider instability of the bosonic D-branes), the long range interaction of the branes goes to zero. This is a consequence of rolling of the tachyon toward its minimum potential. We observe that for the interaction of two D-instantons the conventional long time interaction amplitude restores.

\section{Boundary state and tachyon condensation}

To determine the boundary state, associated with a moving $\mathrm{D} p$-brane in the presence of the tachyon field, we begin with an appropriate sigma-model for the string. This action contains the bulk term, a tachyonic term on the boundary and a velocity term corresponding to the motion of the brane

$$
\begin{aligned}
& S_{\text {bulk }}=-\frac{1}{4 \pi \alpha^{\prime}} \int_{\Sigma} d^{2} \sigma\left(\sqrt{-h} h^{a b} g_{\mu \nu} \partial_{a} X^{\mu} \partial_{b} X^{\nu}\right), \\
& S_{\text {boundary }}=\int_{\partial \Sigma} d \sigma\left(V^{i} X^{0} \partial_{\tau} X^{i}+i U_{\alpha \beta} X^{\alpha} X^{\beta}\right),
\end{aligned}
$$

where $\Sigma$ is the worldsheet of the closed string, exchanged between the branes, $\partial \Sigma$ indicates the boundary of this worldsheet, which can be at $\tau=0$ or $\tau=\tau_{0}$ and the $d$-dimensional spacetime metric is $g_{\mu \nu}=(-1,1, \cdots, 1)$. In addition, we defined $V^{i}=\frac{v^{i}}{2 \pi \alpha^{\prime}}$ where $v^{i}$ is the brane's velocity component along $X^{i}$ direction. The coupling of the tachyon field to the string via integration over the worldsheet boundary has been discussed in [12]. Since it occurs as square in the action, i.e. $T^{2}(X)$, thus in order to produce a Gaussian integral the tachyon profile must be chosen to be linear, $T(X)=a+u_{\mu} X^{\mu}$. The constant $a$ has been shifted away in (2). Note that we also consider the symmetric matrix $U$ to have nonzero elements only along the worldvolume of the D p-brane. The set $\left\{X^{\alpha}\right\}$ specifies the directions along the $\mathrm{D} p$-brane worldvolume and $\left\{X^{i}\right\}$ shows the directions perpendicular to it.

\subsection{The boundary state}

Now consider the mode expansion of the coordinates of the closed string

$$
X^{\mu}(\sigma, \tau)=x_{0}^{\mu}+2 \alpha^{\prime} p^{\mu} \tau+\sqrt{\frac{\alpha^{\prime}}{2}} \sum_{m>0} m^{-1 / 2}\left(x_{m}^{\mu} e^{2 i m \sigma}+\bar{x}_{m}^{\mu} e^{-2 i m \sigma}\right),
$$


where we define $x$ and $\bar{x}$ as combinations of the bosonic modes

$$
\begin{aligned}
& x_{m}=a_{m} e^{-2 i m \tau}+\widetilde{a}_{m}^{\dagger} e^{2 i m \tau}, \\
& \bar{x}_{m}=a_{m}^{\dagger} e^{2 i m \tau}+\widetilde{a}_{m} e^{-2 i m \tau},
\end{aligned}
$$

in which $a_{m}^{\mu}=\frac{i}{\sqrt{m}} \alpha_{m}^{\mu}$ and $a_{m}^{\dagger \mu}=\frac{-i}{\sqrt{m}} \alpha_{-m}^{\mu}$. Similar relations also hold for $\widetilde{a}_{m}^{\mu}$ and $\widetilde{a}_{m}^{\dagger \mu}$. If we interpret the equations (4) as eigenvalue equations [7], the corresponding eigenstate is

$$
|x, \bar{x}\rangle=\prod_{m=1}^{\infty} \exp \left(-\frac{1}{2} \bar{x}_{m} x_{m}-a_{m}^{\dagger} \widetilde{a}_{m}^{\dagger}+a_{m}^{\dagger} x_{m}+\bar{x}_{m} \widetilde{a}_{m}^{\dagger}\right)|v a c\rangle,
$$

where contraction with the metric $g_{\mu \nu}$ is applied implicitly. This state is the boundary state of the closed string due to the bulk term of the string sigma-model without any boundary interaction. Naturally deforming the action by adding non-vanishing boundary contributions, leads to the deformed boundary state

$$
\left|B ; S_{\text {boundary }}\right\rangle=\int[d x d \bar{x}] e^{i S_{\text {boundary }}[x, \bar{x}]}|x, \bar{x}\rangle .
$$

The boundary actions related to the tachyon, $S_{T}$, and the velocity term, $S_{V}$, can be written in terms of modes

$$
\begin{gathered}
S_{T}=i \pi x_{0}^{\alpha} U_{\alpha \beta} x_{0}^{\beta}+i \pi \alpha^{\prime} \sum_{m=1}^{\infty} \bar{x}_{m}^{\alpha} \frac{U_{\alpha \beta}}{m} x_{m}^{\beta}, \\
S_{V}=v^{i} x_{0}^{0} p^{i}+i v^{i} \sum_{m=1}^{\infty}\left(\frac{1}{2}\left(\bar{x}_{m}^{0} x_{m}^{i}+\bar{x}_{m}^{i} x_{m}^{0}\right)-\bar{x}_{m}^{0} a_{m}^{i}-\widetilde{a}_{m}^{i} x_{m}^{0}\right) .
\end{gathered}
$$

From now on we impose a selected direction $X^{i_{0}}$ for the motion of the $\mathrm{D} p$-brane and define $v^{i_{0}}=v$. Substituting (7) and (8) into (6), and also considering the contribution of the bulk action in the boundary, gives the boundary state. The oscillating part of this state is

$$
\left|B_{x}\right\rangle^{\mathrm{osc}}=\prod_{m=1}^{\infty} \frac{1}{\operatorname{det} R_{(m)}} \exp \left(\sum_{m=1}^{\infty} a_{m}^{\dagger} \cdot \mathcal{S}_{(m)} \cdot \tilde{a}_{m}^{\dagger}\right)|0\rangle
$$

where

$$
\left\{\begin{array}{c}
R_{(m) a b}=-2 \Omega_{a b}+\frac{2 \pi \alpha^{\prime}}{m} U_{\alpha \beta} \delta_{a}^{\alpha} \delta_{b}^{\beta}, \\
\Omega_{a b}=-\frac{1}{2} g_{a b}-\frac{1}{2} v\left(\delta^{0}{ }_{a} \delta_{b}^{i_{0}}+\delta^{i_{0}}{ }_{a}{ }^{0}{ }_{b}\right),
\end{array}\right.
$$

and

$$
\mathcal{S}_{(m) \mu \nu}=2\left(R_{(m)}^{-1}\right)_{a b} \delta^{a}{ }_{\mu} \delta^{b}{ }_{\nu}-g_{\mu \nu}
$$

The indices $a$ and $b$ indicate worldvolume and motion directions (i.e. $a, b \in\left\{\alpha, i_{0}\right\}$ ). It is seen that when the velocity $v$ and the tachyon matrix $U$ are zero, there is $\left(R_{(m)}^{-1}\right)_{a b}=g_{a b}$. Hence 
the boundary state (9) reduces to the state for a $\mathrm{D} p$-brane where $X^{\alpha}$ 's, $\alpha=0, \ldots, p$, and $X^{i}$ s, $i=p+1, \ldots, d-1$, obey the Neumann and Dirichlet boundary conditions, respectively [13].

The infinite product in (9) is generated by the path integral. Zeta function regularization can be used to avoid this divergent quantity [14],

$$
\prod_{m=1}^{\infty}\left[\operatorname{det}\left(-2 \Omega+2 \pi \alpha^{\prime} \frac{W}{m}\right)\right]^{-1}=\sqrt{\operatorname{det}(-2 \Omega)} \operatorname{det} \Gamma\left(1-\frac{\pi \alpha^{\prime} W}{\Omega}\right),
$$

where the matrix $W$ is defined by $W_{a b}=U_{\alpha \beta} \delta^{\alpha}{ }_{a} \delta^{\beta}{ }_{b}$.

The zero mode part of the boundary state becomes

$$
\begin{aligned}
\left|B_{x}\right\rangle^{0} & =\frac{T_{p}}{2 \sqrt{\operatorname{det} U}} \int d p^{\alpha} \exp \left(-\frac{1}{4 \pi} P^{T} U^{-1} P\right) \delta\left(x_{0}^{i_{0}}-v x_{0}^{0}-y^{i_{0}}\right) \prod_{i^{\prime} \neq i_{0}} \delta\left(x_{0}^{i^{\prime}}-y^{i^{\prime}}\right) \\
& \times \prod_{\alpha}\left|p_{L}^{\alpha}=p_{R}^{\alpha}\right\rangle \prod_{i^{\prime} \neq i_{0}}\left|p_{L}^{i^{\prime}}=p_{R}^{i^{\prime}}=0\right\rangle\left|p_{L}^{i_{0}}=p_{R}^{i_{0}}=\frac{1}{2} v p^{0}\right\rangle,
\end{aligned}
$$

where the vector $P$ is defined by $P_{\alpha}=v p^{i_{0}} \delta_{\alpha}^{0}-\frac{1}{2} p_{\alpha}$. The momentum dependent exponential term appears due to the presence of the momentum components in the zero mode parts of the boundary actions. Two delta functions indicate the position of the brane. After performing the integration over momenta the matter part of the boundary state takes the form

$$
\begin{aligned}
\left|B_{x}\right\rangle \quad & =\left|B_{x}\right\rangle^{\mathrm{osc}}\left|B_{x}\right\rangle^{0} \\
& =T_{p} \frac{\pi(4 \pi)^{p}}{v^{2}+1 / 2} \prod_{m=1}^{\infty} \frac{1}{\operatorname{det} R_{(m)}} \exp \left(\sum_{m=1}^{\infty} a_{m}^{\dagger} \cdot \mathcal{S}(m) \cdot \widetilde{a}_{m}^{\dagger}\right) \\
& \times \delta\left(x_{0}^{i_{0}}-v x_{0}^{0}-y^{i_{0}}\right) \prod_{i^{\prime} \neq i_{0}} \delta\left(x_{0}^{i^{\prime}}-y^{i^{\prime}}\right)|v a c\rangle,
\end{aligned}
$$

where $|v a c\rangle=|0\rangle_{\alpha}|0\rangle_{\widetilde{\alpha}}|p\rangle$ is written in this form for briefness.

\subsection{Partition function and tachyon condensation}

Since partition function is defined by $\mathcal{Z}=\int D X e^{i S[X]}$, it is obvious that there exists a very natural connection between boundary state and the partition function: the latter is just given by the vacuum amplitude of the boundary state

$$
\mathcal{Z}=\left\langle v a c \mid B ; S_{\text {boundary }}\right\rangle
$$

Therefore, the normalization factors in the Eq. (14) comes from the disk partition function [15] which can also be derived by evaluating the string path integral on a disk

$$
\mathcal{Z}_{\text {disk }}=T_{p} \frac{\pi(4 \pi)^{p}}{v^{2}+1 / 2} \prod_{m=1}^{\infty}\left[\operatorname{det}\left(g_{a b}-v\left(\delta_{a}^{0}{ }_{a} \delta_{b}^{i_{0}}+\delta_{b}^{0}{ }_{b} \delta_{a}^{i_{0}}\right)+\frac{2 \pi \alpha^{\prime}}{m} U_{\alpha \beta} \delta_{a}^{\alpha} \delta_{b}^{\beta}\right)\right]^{-1} \text {. }
$$


Note that the disk diagram in the closed string theory shows a propagating closed string from the boundary of the disk, which then disappears.

Presence of the open string tachyon field as a background field in our case, enables us to study the effect of tachyon condensation on the partition function. In the case at hand where the tachyon profile is linear, studying tachyon condensation equals to sending the elements of the tachyon matrix $U$ to infinity [10].

Here our tachyon matrix possesses all elements along the brane worldvolume. Remember that, $U_{\alpha \beta}$ is a $(p+1) \times(p+1)$ matrix. Without loss of generality let it be a diagonal matrix. We consider condensation of all spatial components of $U$ which can be done one by one for each component or at once for all of them. After successive condensations along the spatial directions of the $\mathrm{D} p$-brane $\left\{X^{\bar{\alpha}} \mid \bar{\alpha}=1,2, \cdots, p\right\}$, which the limit $U_{\overline{\alpha \alpha}} \rightarrow \infty$ is applied, the partition function (16) becomes

$$
\mathcal{Z}_{\text {disk }}=T_{p} \frac{\pi(4 \pi)^{p}}{v^{2}+1 / 2}\left(2 \pi \sqrt{\alpha^{\prime}}\right)^{p} \sqrt{\operatorname{det} U^{\prime}} \prod_{m=1}^{\infty}\left(1-v^{2}+\frac{2 \pi \alpha^{\prime}}{m} U_{00}\right)^{-1}
$$

where $U^{\prime}$ is a new diagonal $p \times p$ tachyon matrix which does not contain the element $U_{00}$. The zeta function regularization, $\prod_{m=1}\left[\operatorname{det}\left(\frac{2 \pi \alpha^{\prime} U^{\prime}}{m}\right)\right]^{-1}=\left(2 \pi \sqrt{\alpha^{\prime}}\right)^{p} \sqrt{\operatorname{det} U^{\prime}}$, has been used in (17). The relation between D-branes' tensions, $T_{p-q}=T_{p}\left(2 \pi \sqrt{\alpha^{\prime}}\right)^{q}$, enables us to interpret (17) as the partition function related to a moving D0-brane with effective tension, $\mathcal{T}_{0}=$ $T_{0} \frac{\pi(4 \pi)^{p}}{v^{2}+1 / 2} \sqrt{\operatorname{det} U^{\prime}}$. This considerable difference with conventional tachyon condensation [10], comes from the momentum dependent exponential factor which exists due to the presence of zero modes in both tachyon and velocity boundary actions. In the absence of the velocity term there is no momentum dependence in partition function and the factor $\frac{1}{\sqrt{\operatorname{det} U}}$ which appears from zero modes in the tachyon action cancels out the factor $\sqrt{\operatorname{det} U}$ which comes from the tachyon condensation in the infinite determinant. However, an additional factor of $\sqrt{\operatorname{det} U}$ appears because of Gaussian integration over momenta and leads to this unusual behavior of partition function after tachyon condensation.

As the next step, performing tachyon condensation along the $X^{0}$-direction in Eq. (17), eliminates the velocity and results in a D-instanton with the partition function of

$$
\mathcal{Z}_{\text {disk }}=T_{(-1)} \frac{\pi(4 \pi)^{p}}{v^{2}+1 / 2} \sqrt{\operatorname{det} U}
$$

In other words, temporal tachyon condensation fixes the D-brane in time as well as eliminates its velocity and fixes it in the space. Generally, temporal condensation on a moving D $p$ brane leads to a stationary instantonic $\mathrm{D} p$-brane (i.e. eliminates the time direction of the worldvolume), and condensation of the spatial components of the tachyon field also reduces the $\mathrm{D} p$-brane dimension. 
Accordingly after tachyon condensation along any spatial direction of the moving $\mathrm{D} p$ brane's worldvolume, its dimension decreases by one in such a manner that after $q$ successive condensations we have a $\mathrm{D}(p-q)$-brane in the presence of a $U_{(p-q+1) \times(p-q+1)}$ tachyon field. The main difference with the usual case is that although the brane's dimension decreases but the effect of the tachyon remains in the root factor.

In the following section, by making use of the boundary state formalism, we compute the interaction amplitude between two D-branes in the closed string channel.

\section{Interaction of the branes}

Since the conformal invariance is preserved in the bulk action (1), and broken on the boundary action (2), [16], the conformal ghosts play role just in bulk and hence their contribution in the boundary state should also be considered. For calculating the interaction amplitude between two D-branes we return to the previous boundary state (14) but restore the integration over momenta. Those give the total boundary state

$$
|B\rangle^{\text {total }}=\left|B_{\mathrm{gh}}\right\rangle\left|B_{x}\right\rangle
$$

To find the interaction amplitude between the $\mathrm{D} p_{1}$ and $\mathrm{D} p_{2}$-branes via exchanging of closed string states, we need closed string propagator which is given by time integral of the closed string Hamiltonian

$$
\begin{aligned}
& D=2 \alpha^{\prime} \int_{0}^{\infty} d t e^{-t H} \\
& H=\alpha^{\prime} p^{\mu} p_{\mu}+2 \sum_{n=1}^{\infty}\left(\alpha_{-n} . \alpha_{n}+\widetilde{\alpha}_{-n} . \widetilde{\alpha}_{n}\right)+(d-2) / 6 .
\end{aligned}
$$

The convention for the indices which will be used in the amplitude is as in the following. The set $\{\bar{i}\}$ shows the directions perpendicular to both branes except $i_{0},\{\bar{u}\}$ is for the directions along both branes except $0,\left\{\alpha_{1}^{\prime}\right\}$ is used for the directions along the $\mathrm{D} p_{1}$-brane and perpendicular to the $\mathrm{D} p_{2}$-brane, and $\left\{\alpha_{2}^{\prime}\right\}$ indicates the directions along the $\mathrm{D} p_{2}$-brane

and perpendicular to the $\mathrm{D} p_{1}$-brane. Since $\left\{\alpha_{1}\right\}$ and $\left\{\alpha_{2}\right\}$ are arbitrary, the position of the branes are not fixed, that is, in our configurations the two branes can be parallel or perpendicular to each other.

\subsection{The interaction amplitude}

The interaction amplitude is given by the overlap of the two boundary state, corresponding to the branes, via the closed string propagator, i.e. $\mathcal{A}=\left\langle B_{1}|D| B_{2}\right\rangle$. After a long calculation 
we obtain

$$
\begin{aligned}
\mathcal{A}=\quad & \frac{\alpha^{\prime} V_{\bar{u}}}{4(2 \pi)^{d} \bar{i}} \frac{T_{p_{1}} T_{p_{2}}}{\left|v_{1}-v_{2}\right|}\left[\operatorname{det} U_{1} \operatorname{det} U_{2}\right]^{-1 / 2} \prod_{m=1}^{\infty}\left[\operatorname{det} R_{(m) 1} \operatorname{det} R_{(m) 2}\right]^{-1} \\
& \times \int_{0}^{\infty} d t\left\{\prod_{m=1}^{\infty}\left(\left[\operatorname{det}\left(1-\mathcal{S}_{(m) 1} \mathcal{S}_{(m) 2}^{T} e^{-4 m t}\right)\right]^{-1}\left(1-e^{-4 m t}\right)^{2}\right)\right. \\
& \times e^{(d-2) t / 6}\left(\sqrt{\frac{\pi}{\alpha^{\prime} t}}\right)^{d_{\bar{i}}} \exp \left(-\frac{1}{4 \alpha^{\prime} t} \sum_{\bar{i}}\left(y_{1}^{\bar{i}}-y_{2}^{\bar{i}}\right)^{2}\right) \frac{1}{\sqrt{\operatorname{det} Q \operatorname{det} G_{1} \operatorname{det} G_{2}}} \\
& \left.\times \exp \left(-\frac{1}{4}\left[E^{T} Q^{-1} E+\sum_{\alpha_{1}^{\prime}}\left[\left(y_{2}^{\alpha_{1}^{\prime}}\right)^{2}\left(G_{1}^{-1}\right)^{\alpha_{1}^{\prime} \alpha_{1}^{\prime}}\right]+\sum_{\alpha_{2}^{\prime}}\left[\left(y_{1}^{\alpha_{2}^{\prime}}\right)^{2}\left(G_{2}^{-1}\right)^{\alpha_{2}^{\prime} \alpha_{2}^{\prime}}\right]\right]\right)\right\} .
\end{aligned}
$$

The matrices $Q, G_{1}$ and $G_{2}$ and the doublet $E$, are defined through their elements as in the following

$$
\begin{gathered}
\left\{\begin{array}{c}
Q_{11}=\frac{\alpha^{\prime} t}{\left(v_{2}-v_{1}\right)^{2}}\left(1+v_{1}^{2}\right)\left(1-v_{2}^{2}\right)-\left[\left(v_{1}^{2}+\frac{1}{2}\right)^{2}\left(U_{1}^{00}\right)^{-1}\right], \\
Q_{22}=\frac{\alpha^{\prime} t}{\left(v_{2}-v_{1}\right)^{2}}\left(1+v_{2}^{2}\right)\left(1-v_{1}^{2}\right)-\left[\left(v_{2}^{2}+\frac{1}{2}\right)^{2}\left(U_{2}^{00}\right)^{-1}\right], \\
Q_{12}=Q_{21}=\frac{\alpha^{\prime} t}{\left(v_{2}-v_{1}\right)^{2}}\left(1+v_{1}^{2}\right)\left(1+v_{2}^{2}\right)\left(1-v_{1} v_{2}\right),
\end{array}\right. \\
\left\{\begin{array}{c}
E_{1}=\frac{i}{v_{2}-v_{1}}\left[y_{2}{ }^{i_{0}}\left(1+v_{1}^{2}\right)^{2}-y_{1}{ }^{i_{0}}\left(1+v_{1} v_{2}\right)\right], \\
E_{2}=\frac{i}{v_{2}-v_{1}}\left[y_{1}{ }^{i_{0}}\left(1+v_{2}^{2}\right)^{2}-y_{2}^{i_{0}}\left(1+v_{1} v_{2}\right)\right],
\end{array}\right.
\end{gathered}
$$

and the nonzero elements of the matrix $G_{1}$ are

$$
\left\{\begin{array}{l}
G_{1 \alpha_{1}^{\prime} \alpha_{1}^{\prime}}=-\alpha^{\prime} t-\frac{1}{4}\left(U_{1}^{\alpha_{1}^{\prime} \alpha_{1}^{\prime}}\right)^{-1} \\
G_{1 \overline{u u}}=-\frac{1}{2} \alpha^{\prime} t-\frac{1}{4}\left(U_{1}^{\overline{u u}}\right)^{-1}
\end{array}\right.
$$

With the exchange $1 \longleftrightarrow 2$ we receive the nonzero elements of $G_{2}$. Note that in $(22)$ there is no sum on the repeated indices $\alpha_{1}^{\prime}$ and $\bar{u}$.

In the interaction amplitude $(19), V_{\bar{u}}$ is the common worldvolume of the branes, and $d_{\bar{i}}$ is the dimension of the directions which are perpendicular to both branes. The infinite product in the second line of (19) shows the effect of the oscillators and conformal ghosts, for analogue of it see the Refs. $[9,17]$. The first exponential and its pre-factor, which are emanated from the directions perpendicular to both branes, indicate the damping of the amplitude due to the distance of the branes. The momenta, which are in the Hamiltonian and zero mode terms in boundary state, leads to the second exponential and the pre-factor of it. The constant factors behind the time integral, somehow show the strength of the interaction which depend on the branes tensions, their velocities and the tachyon fields. Note that the regularization of infinite product in the first line can be done according to (12). Note that the amplitude (19) can be interpreted as cylindrical partition function for closed string, too. 


\subsection{Long time behavior of the interaction amplitude}

One of the interesting features about the interaction amplitude is to study its behavior after long enough times, i.e. $\lim _{t \rightarrow \infty} \mathcal{A}$. In the ordinary cases (i.e. in the absence of background tachyon) massless closed string states dominate in this regime. Here the difference with the conventional interaction amplitudes is the presence of the matrices $Q, G_{1}, G_{2}$ and the doublet $E$, which are functions of time. Therefore, in long distances of the branes in the 26-dimensional spacetime, the closed string tachyon and the massless closed string states (i.e. graviton, dilaton and Kalb-Ramond) contribute to the interaction amplitude as in the following

$$
\begin{aligned}
\mathcal{A}_{0} & =\lim _{t \rightarrow \infty} \mathcal{A} \\
& =\frac{i(-1)^{\left(p_{1}+p_{2}\right) / 2} T_{p_{1}} T_{p_{2}}}{4(2 \pi)^{d_{\bar{i}}}\left(1+v_{1}^{2}\right)\left(1+v_{2}^{2}\right)} \frac{2^{d_{\bar{u}}+1 / 2}}{\left(\alpha^{\prime}\right)^{\left(p_{1}+p_{2}\right) / 2}} \\
& \times\left[\operatorname{det} U_{1} \operatorname{det} U_{2}\right]^{-1 / 2} \prod_{m=1}^{\infty}\left[\operatorname{det} R_{(m) 1} \operatorname{det} R_{(m) 2}\right]^{-1} \\
& \times \int d t\left\{\left(\sqrt{\frac{\pi}{\alpha^{\prime} t}}\right)^{d_{\bar{i}}} \exp \left(-\frac{1}{4 \alpha^{\prime} t} \sum_{\bar{i}}\left(y_{1}^{\bar{i}}-y_{2}{ }^{\bar{i}}\right)^{2}\right)\right. \\
& \left.\times \lim _{t \rightarrow \infty}\left(\frac{e^{4 t}}{t^{1+\left(p_{1}+p_{2}\right) / 2}}+\frac{\operatorname{Tr}\left(\mathcal{S}_{(1) 1} \mathcal{S}_{(1) 2}^{T}\right)-2}{t^{1+\left(p_{1}+p_{2}\right) / 2}}\right)\right\},
\end{aligned}
$$

where $d_{\bar{u}}$ is the dimension of the common worldvolume of the branes. The limit of the exponential and its pre-factor in (23) with respect to $t$ is not important for us because they are related to the position of the branes, while the states of closed string are independent of these positions. The divergent part in the last line, (the first term), corresponds to the tachyonic closed string state. The analogous of this divergent term in the absence of the background tachyon field lacks the decelerating coefficient $1 / t^{1+\left(p_{1}+p_{2}\right) / 2}$ and is usually put away in the literature. It is a deficiency of the bosonic string theory which will be recovered in superstring theory. But the point is that here the time dependence in the denominator slows down this divergence. The other term is related to the contribution of the massless states which also differs from the conventional case, due to the presence of the decelerating factor which makes it to go to zero fast in the limit of long time.

There is a remarkable interpretation for this behavior. Taking into account the open string tachyon as a background field, means working with unstable D-branes. The consequence of this instability is rolling of the tachyon as the system evolves and after long time most of the energy which was localized in the tachyon field transfers to bulk. This is the consequence of decaying of the unstable D-branes into the bulk modes [18]. So, in this picture 
the long time interaction of the D-branes (due to the massless closed string exchange) goes to zero. In other words, after long enough time, there are no D-branes to consider interaction for them. The exchange of the closed string tachyon which is present as a divergent term also has been moderated in this picture. Although this term tends to infinity anyway, its rate of growth is related to the dimension of the branes. Therefore, apart from the tachyonic term which goes to infinity, we can say that the exchange of the massless closed string states cause the D-branes to interact but their contribution decreases in time due to the instability of the D-branes.

The damping of the interaction amplitude by passing the time depends on the branes dimensions. An interesting exception is D-instanton. When two D-instantons interact with each other the factor $1 / t^{1+\left(p_{1}+p_{2}\right) / 2}$ reduces to 1 and hence the ordinary long time amplitude, associated with the massless states, restores. In addition, is the usual divergent term related to the tachyonic closed string state. So we can say that the general interactive behavior of the D-instantons do not change in the presence of background tachyon field.

\section{Conclusions and Summary}

We obtained the boundary state of a closed string, emitted (absorbed) from (by) a moving $\mathrm{D} p$-brane in the presence of the background tachyon field.

The relation between the boundary state and the disk partition function is discussed. The effect of the tachyon condensation on the partition function was studied which shows a spectacular difference from the conventional condensation. Condensation of the tachyon matrix components along any spatial worldvolume directions leads to a partition function corresponding to a lower dimensional moving D-brane with an effective tension which depends on the condensated components of the tachyon field. However, condensation of $U_{00}$ eliminates velocity and as well leads to an instantonic D-brane which is fixed in time. After complete condensation of the tachyon field a D-instanton is obtained.

The interaction amplitude between two D-branes with arbitrary dimensions $p_{1}$ and $p_{2}$ was calculated. Our calculations are valid for the systems that their branes are parallel or perpendicular to each other. The interaction strength between the branes depends on the branes dimensions, their tensions, their relative configuration, the closed string mode numbers, the tachyon matrices and the velocities of the branes.

As a special case, in the large distance interaction of the branes, the contribution of the massless states goes to zero and the divergence part related to the tachyonic state considerably slows down. So the statement that the force associated with the massless 
states is long range would be valid until there is no tachyon background field in the system. This unconventional behavior may be ascribed to the rolling of the tachyon field towards its

minimum potential. This leads to a closed string vacuum without any D-brane at the end of the process and causes the concept of interaction of the D-branes to faint. Interesting point is that in the case of the D-instantons interaction this descension of the long time amplitude jumps to the usual case.

\section{References}

[1] A. Sen, Int. J. Mod. Phys. A20 (2005) 5513-5656.

[2] A. Sen, Int. J. Mod. Phys. A14 (1999) 4061-4078.

[3] D. Kutasov, M. Marino and G. Moore, JHEP 0010 (2000) 045; T. Lee, Phys. Lett. B 520 (2001) 385390.

[4] K. Hashimoto, P. M. Ho and J. E. Wang, Mod. Phys. Lett. A20 (2005) 79-94.

[5] E. Cremmer and J. Scherk, Nucl. Phys. B50 (1972) 222.

[6] M.B. Green and P.Wai, Nucl. Phys.B431 (1994) 131; M. Li, Nucl. Phys. B460 (1996) 351; C. Schmidhuber, Nucl. Phys.B467 (1996) 146; M.B. Green and M. Gutperle, Nucl. Phys. B476 (1996) 484; P. Di Vecchia, M. Frau, I. Pesando, S. Sciuto, A. Lerda and R. Russo, Nucl. Phys.B507 (1997) 259.

[7] J. Callan, C. Lovelace, C. R. Nappi and S. A. Yost, Nucl. Phys. B308 (1988) 221; Nucl. Phys. B288 (1987) 525.

[8] Y. Zhang, Phys. Rev. D63 (2001) 106002; S. P. de Alvis, Phys. Lett. B 505 (2001) 215221; O. Andreev, Nucl. Phys. B598 (2001) 151-168.

[9] H. Arfaei and D. Kamani, Phys. Lett. B452 (1999) 54-60, hep-th/9909167; Nucl. Phys. B561 (1999) 57-76, hep-th/9911146.

[10] T. Lee, Phys. Rev. D64 (2001); G. Arutyunov, A. Pankiewicz and B. Stefanski jr, JHEP 06 (2001) 049.

[11] E. T. Akhmedov, M. Laidlaw and G. W. Semenoff, JETP Lett. 77: 1-6, 2003, PismaZh. Eksp. Teor. Fiz. 77: 3-8, 2003; M. Laidlaw, G. W. Semenoff, JHEP 0311:021, 2003; 
M. Laidlaw, "On a Modification of the Boundary State Formalism in Off-shell String Theory", hep-th/0210270; T. Takayanagi, S. Terashima and T. Uesugi, JHEP 0103:019, 2001.

[12] D. Kutasov, M. Marino and G. Moore, "Remarks on Tachyon Condensation in Superstring Field Theory", hep-th/0010108; JHEP 0010 (2000) 045.

[13] P. Di Vecchia and A. Liccardo, NATO Adv. Study Inst. Ser. C. Math. Phys. Sci. 556 (2000) 1-59.

[14] P. Kraus and F. Larsen, Phys. Rev. D63 (2001) 106004.

[15] E. S. Fradkin and A. A. Tseytlin, Phys. Lett. B163 (1985) 123.

[16] T. Uesugi, "Worldsheet Description of Tachyon Condensation in Open String Theory", hep-th/0302125.

[17] D. Kamani, Mod. Phys. Lett. A15 (2000) 1655-1664, hep-th/9910043.

[18] A. Sen, JHEP 0204 (2002) 048; F. Larsen, A. Naqvi, S. Terashima, JHEP 0302 (2003) 039. 